\title{
Activation of a Subset of Lumbar Spinothalamic Neurons after Copulatory Behavior in Male But Not Female Rats
}

\author{
William A. Truitt, ${ }^{1}$ Michael T. Shipley, ${ }^{2}$ Jan G. Veening, ${ }^{3}$ and Lique M. Coolen ${ }^{1}$ \\ ${ }^{1}$ Department of Cell Biology, Neurobiology, and Anatomy, University of Cincinnati College of Medicine, Cincinnati, Ohio 45267-0521, ${ }^{2}$ Department of \\ Anatomy and Neurobiology, University of Maryland, Baltimore, Baltimore, Maryland 21201, and ${ }^{3}$ Department of Anatomy and Embryology, University of \\ Nijmegen, Nijmegen, 6500HB The Netherlands
}

\begin{abstract}
The precise pathways that convey copulation-related information to forebrain regions activated during male and female sexual behavior are poorly understood. Previous work from our laboratory and others has demonstrated the existence of a spinothalamic pathway that is a candidate to relay information to these areas. This pathway originates from a population of spinothalamic neurons in the lumbar spinal cord containing several neuropeptides including galanin, located in laminas 7 and 10 of the lumbar segments 3 and 4 . To investigate the involvement of these lumbar spinothalamic neurons in conveying copulation-related information, we tested the hypothesis that these cells are activated after ejaculation in male rats and vaginocervical stimulation in female rats. This was assessed using galanin or cholecystokinin as a marker for this subset of spinothalamic neurons and Fos-immunoreactivity as a marker for neuronal activation. The results demonstrated that activation of these spinothalamic neurons is triggered by stimuli associated with ejaculation. Fos induction was specifically associated with ejaculation, because mounts or intromissions did not trigger expression. Moreover, these spinothalamic neurons were not activated by vaginocervical stimulation in female rats. Spinothalamic neurons have generally been associated with signaling pain and temperature information. The present findings demonstrate that a specific subpopulation of spinothalamic neurons signals information associated with ejaculation.
\end{abstract}

Key words: Fos; spinal cord; thalamus; sexual behavior; galanin; spinothalamic

\section{Introduction}

Our current understanding of the neural regulation of sexual behavior in male rodents is based primarily on studies of copulatory behavior after manipulations of specific brain areas (for review, see Meisel and Sachs, 1994). In recent years, the neural circuits underlying male sexual behavior have been investigated using c-Fos expression as a marker for neural activation (for review, see Newman et al., 1997; Pfaus and Heeb, 1997; Veening and Coolen, 1998). A number of these studies have demonstrated the existence of a subcircuit within the larger circuits underlying male sexual behavior, in which neural activation is solely expressed after ejaculation but not after intromissions (Coolen et al., 1996, 1997a,b, 1998; Heeb and Yahr, 1996; Kollack-Walker and Newman, 1997) or other aspects of sexual activity (Coolen et al., 1997b). The subcircuit in which ejaculation-related Fos is expressed consists of small regions within subdivisions of the medial amygdala, the bed nucleus of the stria terminalis, and the medial portion of the parvocellular subparafascicular thalamic nucleus (mSPFp) (Veening and Coolen, 1998). Interestingly, in female rats, Fos-IR after vaginocervical stimulation has a distribution similar to the ejaculation-induced distribution observed in males (Erskine, 1993; Pfaus et al., 1993; Rowe and Erskine,

\footnotetext{
Received Aug. 29, 2002; revised 0ct. 17, 2002; accepted 0ct. 23, 2002.

This research was supported by National Institutes of Health Grant MH60781 (L.M.C.). We acknowledge the contributions of Dr. Michael Lehman, who reviewed a draft of this manuscript and provided many helpful suggestions.

Correspondence should be addressed to William A. Truitt, Department of Cell Biology, Neurobiology, and Anatomy, University of Cincinnati College of Medicine, Vontz Center for Molecular Studies, 3125 Eden Avenue, Cincinnati, OH 45267-0521.E-mail: Bill.Truitt@uc.edu.

Copyright $\odot 2002$ Society for Neuroscience $\quad 0270-6474 / 02 / 220325-07 \$ 15.00 / 0$
}

1993; Tetel et al., 1993; Coolen et al., 1996; Polston and Erskine, 2001). Therefore, we hypothesize that neural activation in this subcircuit is associated with processing of genital sensory signals specifically related to ejaculation or vaginocervical stimulation.

The pathways that convey information from the spinal cord to regions of the forebrain activated after ejaculation or vaginocervical stimulation are unclear. The $\mathrm{mSPFp}$ receives a unique input from a specific subpopulation of spinothalamic neurons in the lumbar spinal cord (Ju et al., 1987). This population of neurons is located in laminas 7 and 10 of lumbar (L) segments 3 and 4 and contains the neuropeptides galanin, cholecystokinin-8 (CCK) (Ju et al., 1987), and enkephalin (Nicholas et al., 1999). Throughout the current paper, this population of lumbar spinothalamic neurons will be referred to as LSt neurons. To test the hypothesis that LSt neurons convey copulation-related information to the mSPFp and possibly other forebrain targets, we investigated whether LSt cells are activated with ejaculation in male rats and vaginocervical stimulation in female rats. Activation of LSt neurons was assessed using Fos-immunoreactivity (IR) as a marker for neural activation and galanin-IR or CCK-IR to identify LSt cells.

\section{Materials and Methods \\ Subjects}

Young adult male $(n=55 ; 250-260 \mathrm{gm})$ and female $(n=19 ; 210-220$ gm) Sprague Dawley rats obtained from Harlan Laboratories (Indianapolis, IN) were housed in same-sex pairs in artificially lighted rooms on a reversed $12 \mathrm{hr}$ light/dark cycle (lights off at 10 A.M.). Food and water were available ad libitum. Stimulus females were ovariectomized and implanted subcutaneously with $5 \% 17-\beta$-estradiol benzoate Silastic capsules. Progesterone (500 $\mu \mathrm{g}$ in $0.1 \mathrm{ml}$ of sesame oil) was injected subcu- 
taneously 4-6 hr before testing to induce sexual receptivity. All procedures were approved by the Animal Care and Use Committee of the University of Cincinnati and conformed to National Institutes of Health guidelines involving vertebrate animals in research.

\section{Behavioral paradigms}

All male and female rats were sexually experienced. Male rats were allowed to copulate during six pretest mating sessions (30 min duration) and were considered sexually experienced after they displayed multiple ejaculations during the last two mating sessions. The female rats were bilaterally ovariectomized 2 weeks before testing. Ovariectomized rats were injected subcutaneously with $5 \mu \mathrm{g}$ of $17-\beta$-estradiol benzoate 48 and $24 \mathrm{hr}$ before testing and with $500 \mu \mathrm{g}$ of progesterone subcutaneously 4-6 hr before testing, to induce sexual receptivity. One week before testing, females received hormone treatment and mating experience with sexually experienced male partners. All testing of experimental groups was performed $4 \mathrm{hr}$ after onset of the dark period, in a rectangular mating arena $(60 \times 45 \times 50 \mathrm{~cm})$, under dim red illumination. Stimulus partners were removed after completion of the mating tests, whereas experimental animals remained in the test cage for an additional $60 \mathrm{~min}$ until they were killed.

\section{Experiment 1: activation of LSt neurons in male rats}

Male rats were divided into five experimental groups and one control group to study induction of Fos-IR after different components of sexual behavior. The male control group consisted of males killed immediately after removal from their home cage. The first experimental group consisted of males that interacted with an anestrous female (AN; $n=9)$. The second group consisted of males that only displayed mounts $(\mathrm{MN} ; n=$ 9). To prevent occurrence of intromissions, the anogenital regions of the stimulus females were covered with masks. Females were removed after an average display of 25.4 mounts. The third group consisted of males displaying mounts and intromissions but no ejaculations (IM; $n=10$ ). Females were removed after an average of 13.1 intromissions. In the fourth group, males were tested until one ejaculation was achieved (E1; $n=11$ ). Finally, the fifth group of males was tested until two ejaculations were achieved (E2; $n=7)$.

Experiment 2: neural activation after 8-hydroxy-2-(di-Npropylamino) tetralin-induced ejaculation

The purpose of experiment 2 was to investigate neural activation of lumbar spinal cord cells in male rats that displayed minimal sexual activity before ejaculation. In experiment 2, male rats were treated with 8-hydroxy-2-(di- $N$-propylamino) tetralin (8-OH-DPAT), which decreases the number of mounts and intromissions before ejaculation (Ahlenius et al., 1981). Experienced male rats received injections of 8-OH-DPAT ( $0.8 \mathrm{mg} / \mathrm{kg}$, s.c.; Sigma, St. Louis, MO) $30 \mathrm{~min}$ before being placed in the testing arena. Males either remained in the testing arena without exposure to a female (DPC; $n=3$ ) or were paired with receptive females for one copulatory series including one ejaculation (DP1E; $n=$ 3). Only male rats that displayed ejaculation within three intromissions were included in this experiment.

\section{Experiment 3: activation of LSt neurons in female rats}

The experimental female groups were divided based on the sexual activity the females received from the male partners. Control females $(n=6)$ received a male partner that did not display sexual behavior, and consequently these females did not display lordosis behavior. The first female experimental group consisted of females that received mounts but no intromissions ( $\mathrm{MN} ; n=4)$. To prevent occurrence of intromissions, the genital regions of the male partners were masked. Stimulus males were removed after display of 12-15 mounts. The second experimental group of females received $6-15$ intromissions $(6-15 \mathrm{IM} ; n=8)$. The final group included females that received 16-25 intromissions (16-25IM; $n=7)$. Females in the latter two groups also received mounts and ejaculations. Ejaculations were counted as intromissions.

Experiment 4: activation of LSt cells after female pacing behavior Previous studies have reported that sexual behavior is rewarding for female rats only if the female is allowed to pace the behavior (Paredes and
Vazquez, 1999). Therefore, the purpose of experiment 4 was to investigate whether vaginocervical stimulation received during paced behavior results in activation of LSt cells in female rats. Two groups of female rats were ovariectomized and treated with estrogen and progesterone as described above. One group of females (paced; $n=6$ ) was placed in a test cage containing a divider to create two separate areas within the test cage; the space between the bottom of the cage and the divider permitted the female to move freely from one area to the other. In contrast, the male was confined to one area; this paradigm allowed the female to pace the behavior. Females received an average of 19.8 intromissions in this paced setting. A second group of females (nonpaced; $n=6$ ) received an average of 20.3 intromissions in the test cage without the divider, similar to the testing described in experiment 3.

\section{Experiment 5: hemisection}

To verify that axons from an identified group of galanin-containing spinothalamic neurons were indeed terminating in the mSPFp, spinal cords of adult male $(n=3)$ and female $(n=2)$ rats were transected unilaterally at lumbar level L1. The female rats were ovariectomized as described above and received subcutaneous estrogen implants (5\% in cholesterol). Spinal cords were fixed in a stereotaxic apparatus, and laminectomy was performed to expose the spinal cord. After removal of dura mater, a microdissection knife was lowered at the midline of $\mathrm{L} 1$ and spinal cord was destroyed unilaterally. The spinal cord was covered with gel foam, and the muscle tissue and incision were closed with sutures and wound clips. Animals were killed $14 \mathrm{~d}$ after surgery, and spinal cord and brain were sectioned.

\section{Perfusion, tissue processing, and immunocytochemistry}

At $1 \mathrm{hr}$ after the end of the behavioral test, animals were anesthetized using sodium pentobarbital $(200 \mathrm{mg} / \mathrm{kg}$, i.p.) and perfused transcardially with $100 \mathrm{ml}$ of $0.9 \%$ saline, followed by $500 \mathrm{ml}$ of $4 \%$ paraformaldehyde in $0.1 \mathrm{M}$ sodium phosphate buffer (PB), $\mathrm{pH}$ 7.3-7.4. Brain and spinal cords were removed and postfixed for $1 \mathrm{hr}$ at room temperature in the fixative. Coronal sections of thoracic ( $\mathrm{T})$, lumbar, and sacral (S) spinal cord (T10-S4) were cut at $35 \mu \mathrm{m}$ on a Microm freezing microtome (Richard Allen, Kalamazoo, MI) and collected in 12 parallel series in cryoprotectant solution (30\% sucrose and $30 \%$ ethylene glycol in $0.1 \mathrm{M}$ PB) (Watson et al., 1985). In addition, coronal sections (35 $\mu \mathrm{m})$ through thalamus were cut and stored in four parallel series.

All incubations were performed at room temperature with gentle agitation. Series of spinal cord sections (420 $\mu \mathrm{m}$ apart) were extensively rinsed in PBS between incubations. Free-floating sections were blocked with $1 \% \mathrm{H}_{2} \mathrm{O}_{2}$ for $10 \mathrm{~min}$ at room temperature and then soaked for $1 \mathrm{hr}$ in incubation solution (PBS containing $0.1 \%$ bovine serum albumin and $0.4 \%$ Triton X-100). Next, sections were incubated overnight with a primary antiserum in incubation solution to recognize Fos [polyclonal anti-Fos antiserum (SC52) raised in rabbit; 1:10,000; Santa Cruz Biotechnology, Santa Cruz, CA]. Subsequently, sections were exposed for 60 min to biotin-conjugated donkey anti-rabbit IgG (1:400 in incubation solution; Jackson ImmunoResearch, West Grove, PA) and for $60 \mathrm{~min}$ to avidin-biotin complex $(\mathrm{ABC})$-horseradish peroxidase (ABC Elite, 1:1000 in PBS; Vector Laboratories, Burlingame, CA). The peroxidase complex was visualized by exposure for $10 \mathrm{~min}$ to a chromogen solution containing $0.02 \% \quad 3,3^{\prime}$-diaminobenzidine tetrahydrochloride (DAB; Sigma) enhanced with $0.02 \%$ nickel sulfate in $0.1 \mathrm{M} \mathrm{PB}$ with hydrogen peroxide $(0.015 \%)$ to produce a blue-black reaction product. Extensive washing in $0.1 \mathrm{M}$ PBS terminated the reaction. The sections were then blocked with $1 \% \mathrm{H}_{2} \mathrm{O}_{2}$ for $10 \mathrm{~min}$ at room temperature and incubated overnight with primary antiserum in incubation solution to recognize galanin (polyclonal anti-galanin antiserum raised in rabbit, 1:80,000; PenLabs, San Carlos, CA) or CCK (polyclonal anti-CCK raised in rabbit, 1:30,000; Chemicon, Temecula, CA). Next, sections were processed using the same avidin-biotin immunoperoxidase procedure described above with the second chromogen reaction performed without nickel sulfate, to produce a brown reaction product. Sections through the spinal cord and thalamus of animals with hemisections were processed for galanin-IR using DAB as described above. Sections were mounted on Superfrost Plus glass slides (Fisher, Pittsburgh, PA) and coverslipped 


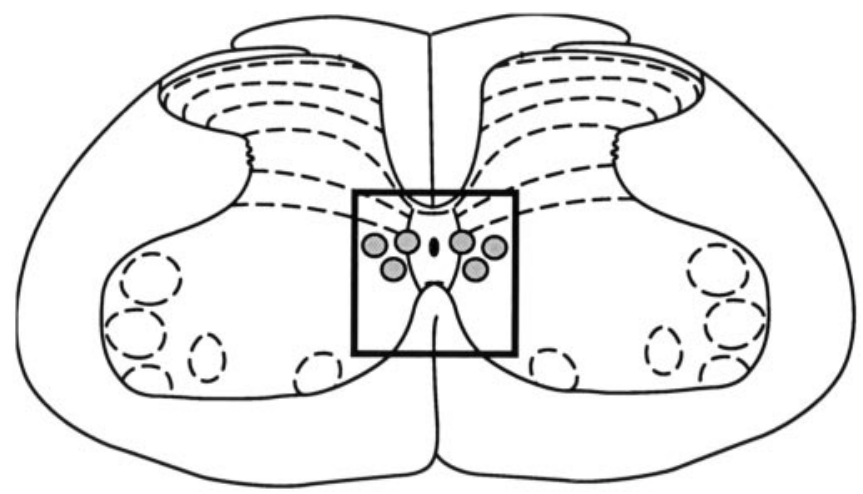

Figure 1. Schematic drawing of $L 4$ illustrating the area of analysis $(800 \times 800 \mu \mathrm{m})$ for Fos-IR and activated LSt cells. Gray circles indicate the approximate location of LSt cells. This figure was modified from Paxinos and Watson (1998).

with DPX (Electron Microscopy Sciences, Fort Washington, PA). Immunocytochemical controls included omission of primary antibodies for Fos, galanin, or CCK.

\section{Data analysis}

Sexual behavior. The number of mounts, the number of intromissions, and the total duration of the tests were recorded for analysis of copulatory behavior. Numbers of mounts and intromissions as well as duration of test were compared using a one-way ANOVA. Post hoc comparisons were made using the Scheffé test, with an $\alpha$ of 0.05 required for rejection of the null hypothesis.

Immunocytochemistry. Analysis of labeling for galanin, CCK, or Fos and dual labeling for Fos and galanin or CCK was performed on a Leica (Deerfield, IL) light microscope at 10-40× magnification. Neurons single labeled for galanin or CCK were determined by brown staining of cytoplasm with a nucleus devoid of blue-black (Fos) reaction product. Neurons with only blue-black nuclei were considered single labeled for Fos. Cells that contained both stained nucleus (blue-black) and cytoplasm (brown) were considered dual labeled. Single-labeled Fos cells were counted in a standard area $(800 \times 800 \mu \mathrm{m})($ Fig. 1) surrounding the central canal, encompassing lamina 10 and medial portions of lamina 7 at levels L3 and L4; this area outlines the location of LSt cells. At each level, Fos cells were counted bilaterally in two sections stained for Fos and galanin. The four counts in L3 and L4 were averaged for individual rats, and group means were calculated. Dual labeling was analyzed within the same area in sections stained for Fos and galanin or Fos and CCK and was expressed as the percentage of galanin or CCK cells that expressed Fos; group means were based on the percentage of double-labeled cells for individual rats. To determine differences between groups, a one-way ANOVA and post hoc comparisons using the Scheffé test were performed, both with a 0.05 level of significance. A Spearman's rank correlation test was performed to determine whether a correlation exists between behavior (duration, number of mounts, or number of intromissions) and the percentage of double-labeled neurons, using a 0.05 level of significance.

Data presentation. Digital color images were imported into Adobe Photoshop 6.0 software (Adobe Systems, San Jose, CA). Images were not adjusted or altered in any way, except for occasional adjustment of brightness.

\section{Results}

Experiment 1: activation of LSt neurons in male rats

Activation of LSt neurons was investigated in male rats after different elements of sex behavior. Table 1 summarizes the behavioral data (numbers of mounts and intromissions and total test duration) for all male experimental groups. Overall, there were few differences in the behavioral parameters between groups. Specifically, the number of mounts and intromissions did not significantly differ between groups. However, not surprisingly,
Table 1. Summary of male sex behavior by group

\begin{tabular}{lllc}
\hline Males & Mean mounts & Mean intromissions & Mean duration (sec) \\
\hline $\mathrm{AN}(n=9)$ & & & $677.6 \pm 66.2$ \\
$\mathrm{MN}(n=9)$ & $25.4 \pm 2.4$ & & $726.1 \pm 75.5$ \\
$\mathrm{IM}(n=10)$ & $17.9 \pm 2.5$ & $13.1 \pm 1.9$ & $473.6 \pm 57.9$ \\
E1 $(n=11)$ & $29.0 \pm 2.7$ & $15.8 \pm 1.1$ & $663.1 \pm 76.7$ \\
E2 $(n=7)$ & $28.8 \pm 4.2$ & $19.4 \pm 2.3$ & $1029.0 \pm 102.6^{*}$ \\
\hline
\end{tabular}

The mean \pm SEM for number of mounts and intromissions as well as the mean \pm SEM time spent with a female are shown. ${ }^{*} p<0.05$, different from IM and E1.
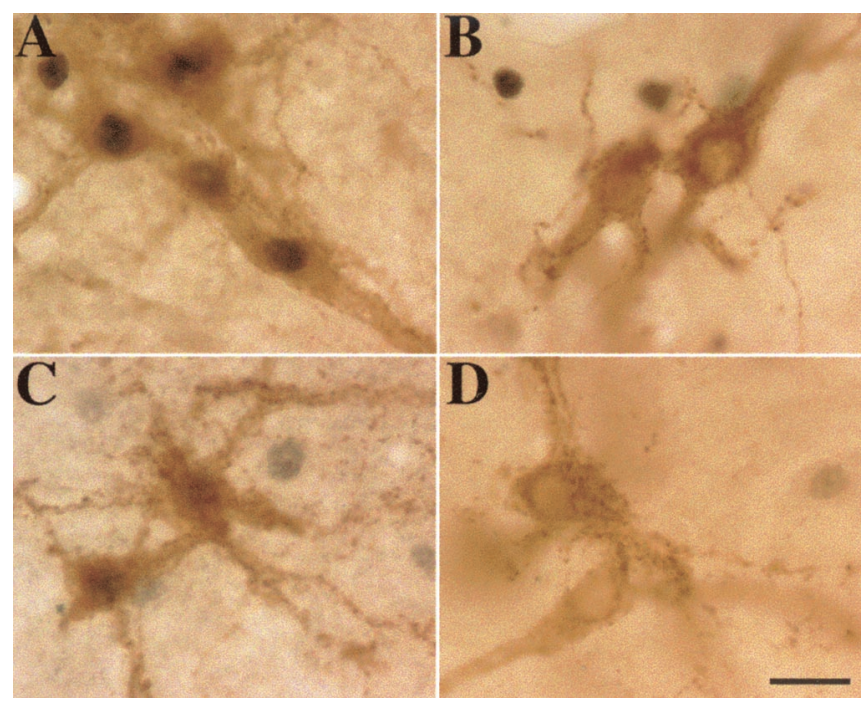

Figure 2. Neural activation of LSt neurons in male but not female spinal cord. A, Galanin neurons are Fos positive in a representative male after two ejaculations. $B$, Lack of colocalization of Fos and galanin in a male rat after intromissions but no ejaculation. C, Colocalization of galanin and Fos in an 8-OH-DPAT-treated male rat after one ejaculation. D, Lack of colocalization of Fos and galanin in a female rat after ejaculation by her male partner. Scale bar, $20 \mu \mathrm{m}$.

test duration was longer for E2 males than for E1 or IM males $(p<0.04)$.

In all males, galanin-IR neurons were observed in laminas 7 and 10 of L3 and L4 (Fig. 2), and the mean number of galanin-IR neurons was similar for all groups, ranging from $28.14 \pm 3.39$ to $36.30 \pm 3.60$ (mean \pm SEM). The percentage of galanin neurons that expressed Fos was significantly higher in males that ejaculated $(83.39 \%)$ compared with males that displayed other elements of sexual behavior (6.95\%) (Fig. 3A) (Scheffé test; $p<$ $0.007)$. Two ejaculations did not result in an additional increase in the percentage of Fos-positive galanin-IR cells. Males engaged in pursuit of an anestrous female or mounting behavior alone failed to display any activation of galanin-IR neurons. In addition, intromissions alone did not result in a significant increase in Fos expression in galanin neurons (Fig. 3A). However, 3 of 10 males in the IM group did display some colocalization of galanin and Fos. The percentages of double labeling in these animals were variable $(27.5,81.8$, and $90.2 \%)$ and were not correlated with the number of mounts or intromissions in these individuals (Spearman's rank correlation; mounts, $p=0.134$; intromissions, $p=$ 0.372 ). Curiously, these IM males displayed atypical mounting behavior. In contrast to normal mounts, during which males display pelvic thrusting but no insertion, mounts in these three males resembled the onset of ejaculatory behavior, attaining a horizontal position atop the female but not displaying thrusting.

Because LSt neurons coexpress galanin and CCK (Ju et al., 1987), mating-induced activation of these neurons was also assessed using CCK-IR as a marker. The mean \pm SEM number of 

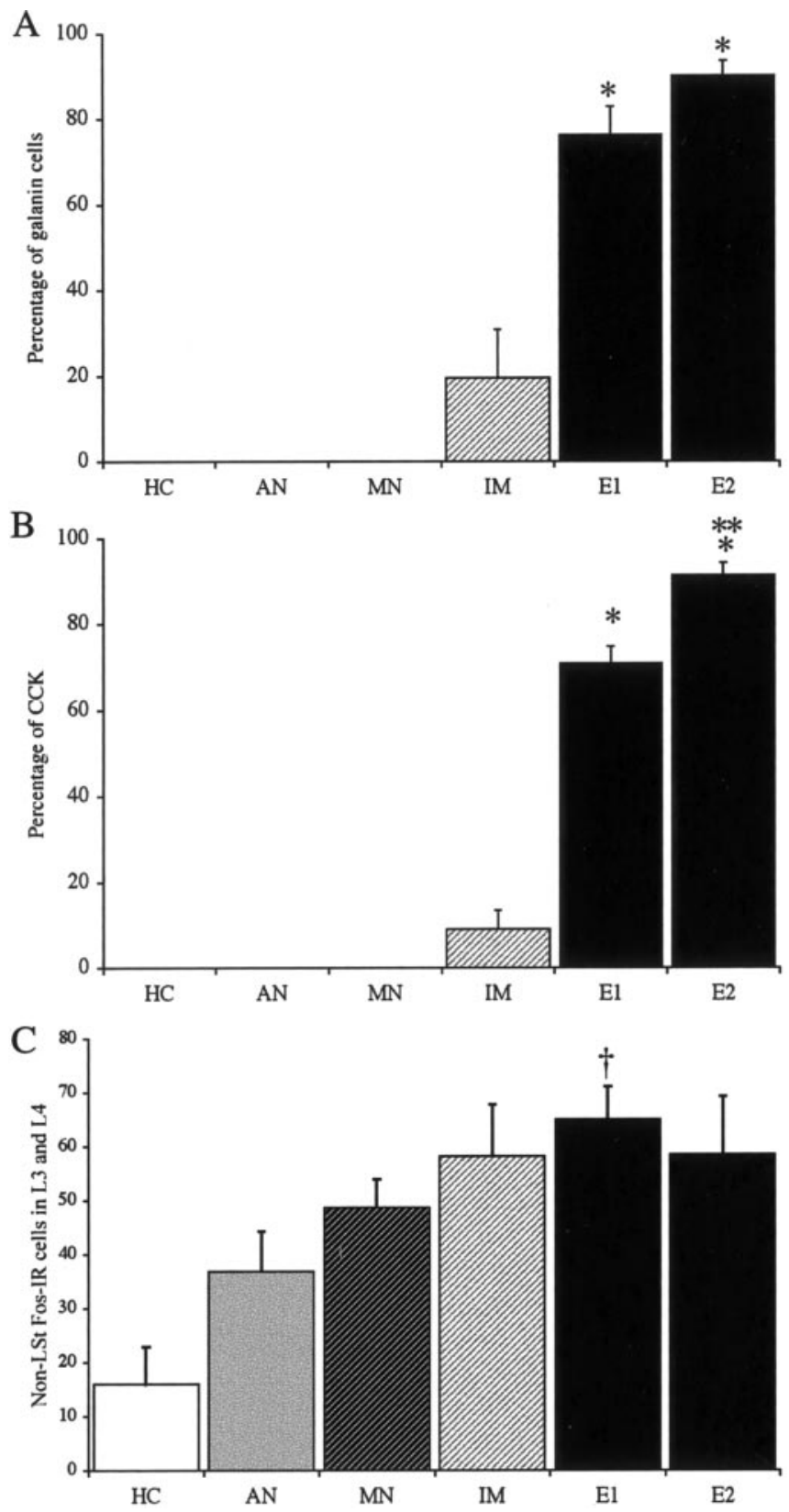

Figure 3. Fos-IR in LSt and non-LSt cells in the $L 3$ and $L 4$ of male rats. $A$, Percentage of galanin-IR neurons that are Fos-IR in male rats. Mean percentages \pm SEM of galanin-IR cells that are Fos-IR per behavioral group ( $H C$, home cage controls; $A N$, males that were placed with an anestrous female; $M N$, males that only mounted; $I M$, males that mounted and displayed intromissions; $E 1$, males that displayed copulatory behavior including 1 ejaculation; $E 2$, males that displayed copulatory behavior including 2 ejaculations). $B$, Percentage of (CK-IR neurons that are Fos-IR in male rats. Mean percentages \pm SEM of CCK-IR cells that are Fos-IR per behavioral group. C, Fos-IR in nongalanin neurons. Mean numbers of Fos-IR in the area surrounding the central canal in $\mathrm{L} 3-\mathrm{L} 4$ per behavioral group in male rats. ${ }^{*} p<0.0001$, significantly different from $\mathrm{HC}$ and different from all other groups; ${ }^{* *} p=0.0003$, different from E1; ${ }^{\dagger} p=0.015$, greater than home cage controls.

CCK-IR neurons did not significantly differ between groups and ranged from $27.71 \pm 3.86$ to $42.75 \pm 6.81$. Furthermore, the average number of CCK-IR neurons did not differ from the average number of galanin-IR neurons $(35.02 \pm 1.78$ and $34.20 \pm$ 1.51 , respectively). As was the case for the galanin-IR cells, there was a significant increase in the percentage of CCK-IR cells expressing Fos after ejaculation (78.68\%) but not after other com-

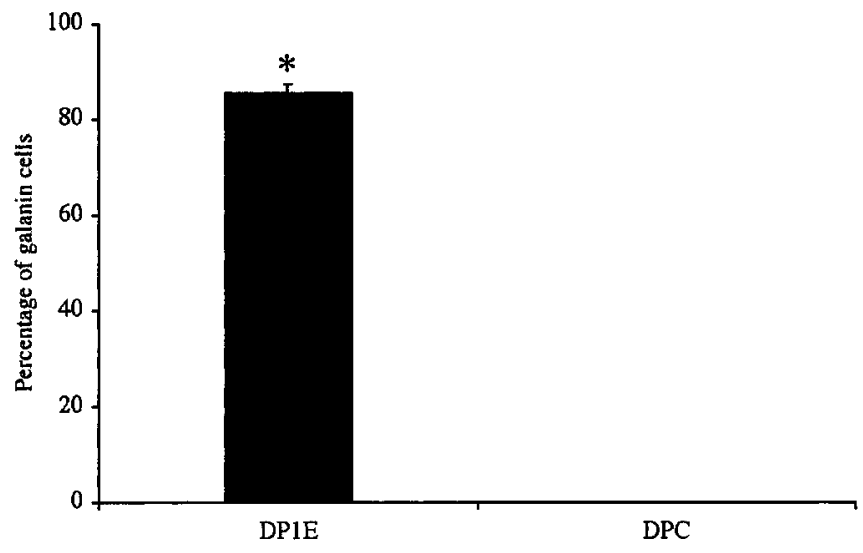

Figure 4. Percentage of galanin-IR neurons that are Fos-IR after 8-OH-DPAT treatment Mean percentages \pm SEM of galanin-IR neurons expressing Fos in rats injected with $0.8 \mathrm{mg} / \mathrm{kg}$ 8-OH-DPAT after copulation to one ejaculation (DP1E; $n=3$ ) or without exposure to a female $(D P C ; n=3) ;{ }^{*} p \leq 0.0001$

ponents of male sexual behavior $(4.46 \% ; p \leq 0.0001)$ (Fig. $3 B$ ). However, in contrast to the galanin-IR neurons, the percentage of the CCK-IR neurons that were Fos-IR was significantly greater in rats with two ejaculations than rats with one ejaculation (Scheffé test; $p=0.015)$. No AN, MN, or IM males displayed activation of CCK-IR cells, with one exception: the same three IM males that showed Fos expression in galanin cells also has Fos-positive CCK cells. However, the percentage of CCK-IR neurons that were Fos-IR in the IM group was not significantly different from the $\mathrm{HC}, \mathrm{AN}$, or MN groups.

Male sexual behavior also resulted in an increase in Fos-IR in neurons that did not contain galanin (non-LSt) in L3 and L4 (Fig. $3 C)\left(F_{(5,45)}=4.16 ; p<0.003\right)$. Rats that copulated to one ejaculation had significantly greater Fos-IR than home cage controls (Scheffé test; $p=0.017$ ). However, this activation was not related specifically to expression of ejaculation, because no differences in numbers of Fos-IR neurons were detected between experimental groups. Finally, in agreement with previous reports (Coolen et al., 1996), Fos-IR in the mSPFp was only induced after one or two ejaculations, and not after intromissions or mounts (L. M. Coolen, J. G. Veening, D. W. Petersen, and M. T. Shipley, unpublished observations).

\section{Experiment 2: activation of LSt neurons after 8-OH-DPAT-induced ejaculation}

All DP1E rats ejaculated in three or fewer intromissions, with one rat ejaculating on the first mount. Thus, the average numbers of mounts $(2.33 \pm 1.20)$ and intromissions $(1.33 \pm 0.67)$ were lower compared with $\mathrm{E} 1$ males in experiment 1 . As in experiment 1 , the mean number of galanin-IR neurons did not differ between groups $(33.67 \pm 4.06$ and $33.33 \pm 5.53)$, and the mean number of galanin-IR neurons counted in this experiment did not differ from experiment 1. Colocalization of Fos-IR and galanin-IR neurons was observed in all DP1E males (Fig. 4), with $85.55 \pm$ $1.76 \%$ of galanin-IR neurons containing Fos-IR. Treatment with 8-OH-DPAT alone (DPC group) did not result in activation of galanin neurons.

\section{Experiment 3: absence of Fos expression in LSt neurons in female rats}

Table 2 summarizes the behavioral data for female rats. The experimental groups only differed significantly in the duration of test, with both groups of females receiving intromissions having 
Table 2. Summary of female sex behavior by group

\begin{tabular}{lrlc}
\hline Females & Mean mounts & Mean intromissions & Mean duration (sec) \\
\hline Control $(n=6)$ & & & \\
$\operatorname{MN}(n=4)$ & $15.0 \pm 1.9$ & & $420.0 \pm 114.0^{*}$ \\
$6-15 \mathrm{IM}(n=8)$ & $5.7 \pm 2.2$ & $10.9 \pm 1.1^{*}$ & $772.5 \pm 104.2^{*}$ \\
$16-25 \mathrm{IM}(n=7)$ & $8.3 \pm 2.1$ & $20.6 \pm 1.6$ & $1748.6 \pm 219.7^{* *}$ \\
\hline
\end{tabular}

The mean \pm SEM for number of mounts and intromissions received by the female as well as the mean \pm SEM time spent with a male are shown. ${ }^{*} p \leq 0.05$, significant difference compared with $16-25 \mathrm{IM} ;{ }^{* *} p \leq 0.004$, significant difference compared with $6-15 \mathrm{IM}$.

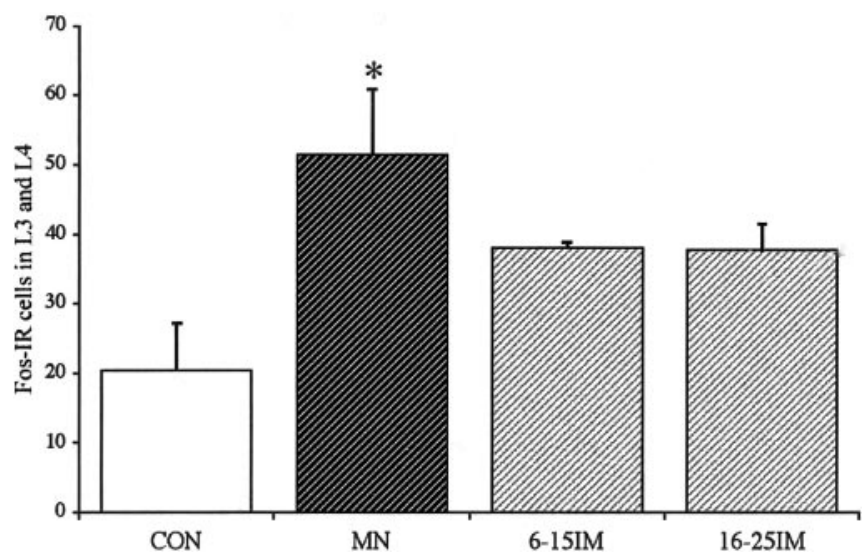

Figure 5. Fos-IR in non-LSt neurons in the $L 3$ and $L 4$ in female rats. Mean \pm SEM numbers of Fos-IR in the area surrounding the central canal in the $L 3$ and $L 4$ in female control rats (CON; $n=6)$, females receiving mounts only (MN; $n=4)$, females receiving $6-15$ intromissions including mounts and ejaculations $(6-15 I M ; n=8)$, and females receiving $16-25$ intromissions $(16-25 I M ; n=7) .{ }^{*} p=0.04$, significantly different from CON (Scheffé test).

Table 3. Summary of paced and nonpaced female sex behavior

\begin{tabular}{llll}
\hline Females & Mean mounts & Mean intromissions & Mean duration (sec) \\
\hline Paced $(n=6)$ & $38.3 \pm 10.7$ & $19.8 \pm 1.0$ & $1959.2 \pm 311.5$ \\
Nonpaced $(n=6)$ & $35.0 \pm 7.6$ & $20.3 \pm 0.3$ & $3233.5 \pm 966.0$ \\
\hline
\end{tabular}

The mean \pm SEM for number of mounts and intromissions received by the female as well as the mean \pm SEM time spent with a male are shown. No significant differences were detected between paced and nonpaced females.

significantly greater test duration than other groups (Scheffé test; $p<0.004)$. The mean numbers of galanin-IR cells did not differ between groups (ranging from $24.33 \pm 1.86$ to $37.67 \pm 6.17$ ), and the mean number of galanin-IR cells in females $(30.17 \pm 1.34)$ did not differ from that of males $(34.12 \pm 1.37)$. Unlike males, female rats showed no colocalization of Fos-IR and galanin-IR or CCK-IR in any group. However, in agreement with previous studies (Coolen et al., 1997b), Fos-IR was observed in the mSPFp of females receiving intromissions but not in females that received mounts without intromissions (Coolen, Veening, Petersen, Shipley, unpublished observations).

Female sexual behavior did result in increased Fos-IR in nonLSt neurons of the L3 and L4 (Fig. 5) $\left(F_{(3,21)}=3.598\right.$; Scheffé test; $p=0.0306)$. Female rats receiving mounts had greater Fos-IR than control females (Scheffé test; $p=0.04$ ); however, Fos-IR in females receiving intromissions was not significantly greater than that seen in control females.

\section{Experiment 4: absence of Fos expression in LSt neurons in pacing females}

Table 3 summarizes the behavioral data for pacing rats. Vaginocervical stimulation resulting from 17 to 22 intromissions from the male partner (including up to three ejaculations) did not induce Fos expression in LSt neurons of females that were al-
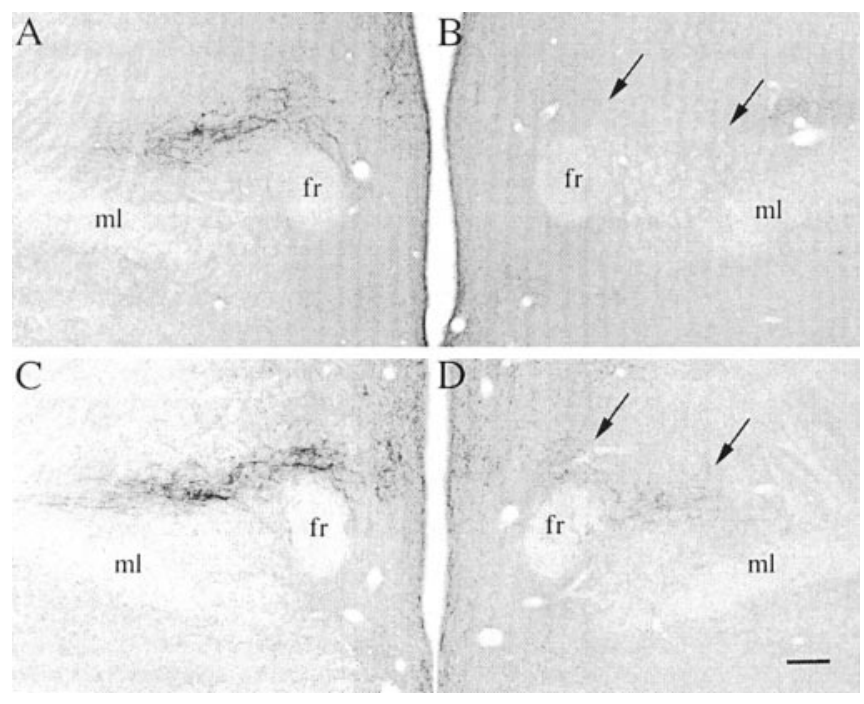

Figure 6. Photomicrographs of coronal sections illustrating decreased galanin-IR fibers in the mSPFp after hemisection at level $L 1$ in male $(A, B)$ and female $(C, D)$ rats. $B, D$, The mSPFp ipsilateral to the transected side of the spinal cord is devoid of galanin-IR, as indicated by arrows. fr, Fasciculus retroflexus; $m l$, medial lemniscus. Scale bar, $200 \mu \mathrm{m}$.

lowed to pace. In agreement with experiment 3, Fos expression in LSt cells was not observed in nonpacing females. Furthermore, pacing did not increase Fos-IR in non-LSt cells in L3 and L4, with pacing females displaying $56 \pm 10.67$ Fos-IR cells (mean \pm SEM) and nonpaced females displaying $54 \pm 4.49$ Fos-IR cells $($ mean \pm SEM $)$.

\section{Experiment 5: hemisections}

In both male and female rats, spinal cord hemisection at L1 severely reduced galanin-IR fiber labeling in the ipsilateral mSPFp (Fig. 6) suggesting that the axons of galanin-IR cell bodies in L3 and L4 cross the midline caudal to L1. One male and one female regained motor control of the affected hindlimb within the 2 week recovery period, indicating an incomplete hemisection. Indeed, in these two animals, galanin-IR was only slightly reduced.

\section{Discussion}

Spinothalamic neurons have been associated with signaling pain and temperature information (Tracey, 1995). Here we demonstrate that a specific subpopulation of spinothalamic neurons composed of galanin- and CCK-IR LSt neurons is involved in the signaling of information pertaining to ejaculation. In both male and female rats, these LSt neurons project to the MSPFp, a brain region that has been reported previously to express Fos after ejaculation or vaginocervical stimulation in male and female rodents, respectively (Pfaus et al., 1993; Kollack-Walker and Newman, 1995, 1997; Coolen et al., 1996; Heeb and Yahr, 1996). However, the present findings demonstrate that the LSt neurons are only activated in male rats with ejaculation and not with vaginocervical stimulation in female rats. These results suggest that this spinothalamic pathway is involved in the processing of information selectively related to ejaculation.

Activation of LSt neurons is triggered by stimuli associated with ejaculation and not other aspects of sexual behavior, because Fos expression is observed with ejaculation but not mounts or intromissions. This raises the question: What is unique about stimuli associated with ejaculation? Intromissions require penile erection, which, in turn, includes relaxation of smooth muscles and activation of striated muscles involved in flips, engorgement 
of the glans penis, and straightening of the penis (for review, see Meisel and Sachs, 1994). Intromission also includes superficial stimulation of the penis during insertion into the vagina. Animals that ejaculate have two additional phases, emission and expulsion. Emission consists of moving seminal fluids into the proximal urethra and involves sympathetically mediated contraction of the vas deferens and epididymis and parasympathetically mediated contractions of the prostate (Newman et al., 1982; DeGroat and Steers, 1990). The expulsion phase of ejaculation is the forceful emptying of the urethral contents and is accompanied by rhythmic bursting of the ischiocavernosus and bulbocavernosus muscles as well as urethral dilation and contractions of the bladder neck, internal urinary sphincter, and anal sphincter (Newman et al., 1982; DeGroat and Steers, 1990; Meisel and Sachs, 1994). Therefore, afferent information accompanying ejaculation may be composed of somatosensory, visceral sensory, and proprioceptive signals. In addition, afferent inputs may include noxious signals.

It is unclear whether activation of LSt neurons is induced by stimuli associated with events before, during, or after ejaculation. The possibility that activation is associated with events immediately before ejaculation is supported by observations that Fos was induced in LSt neurons in 3 of the 10 rats displaying intromissions but not ejaculation. In particular, these males displayed atypical mounting behavior that resembles the onset of ejaculatory behavior and is often immediately followed by a display of ejaculation (W. A. Truitt and Coolen, unpublished observations). Thus, it is possible that activation of LSt neurons results from summation of sexual stimulation required for triggering ejaculatory reflexes. However, it is also possible that these three IM males ejaculated in the absence of a female. Because we did not continue to observe the males closely after removal of the female, such an event may have been overlooked.

Although activation of LSt neurons may result from a summation of stimuli preceding ejaculation, results from experiment 2 clearly demonstrate that summation of sexual activity normally preceding ejaculation is not required to elicit Fos expression. 8-OH-DPAT-treated males had similar proportions of activated LSt neurons but had minimal sexual activity before ejaculation compared with rats in experiment 1. Likewise, 8-OH-DPATtreated males with minimal sexual activity before ejaculation express Fos in patterns similar to normal ejaculating males in the mSPFp and in other brain structures in which Fos is associated with ejaculation (Coolen et al., 1997b).

In addition to the hypothesis that LSt activation occurs before ejaculation, it is possible that activation of LSt cells occurs during ejaculation. For example, LSt neurons may receive inputs associated with emission or associated with expulsion of the seminal fluids. Moreover, activation of LSt neurons may be associated with events that occur after ejaculation such as autogrooming or sexual inactivity.

The location of LSt neurons is well suited to receive ejaculation-related inputs. Ejaculation-related information is conveyed by several nerves, including the pudendal, hypogastric, and pelvic nerve (DeGroat and Steers, 1990; Meisel and Sachs, 1994; Veening and Coolen, 1998). The majority of the afferent fibers in these nerves enter the spinal cord at levels T12 to L2 (hypogastric) and L6 to S3 (pelvic and pudendal) (DeGroat and Steers, 1990). However, some primary afferents project in Lissauer's tract rostral and caudal to the entry zone. These afferents extend rostrally as far as the rostral part of L3, where they tend to concentrate in the medial portion of Lissauer's tract (Nadelhaft and Booth, 1984). Nadelhaft and Booth (1984) have suggested that this rostrally directed pathway may be involved in transmission of sensory information from the periphery. The LSt neurons are therefore ideally situated to receive ejaculation-related inputs and possibly integrate information arising from any or all of these nerves. Furthermore, LSt neurons are located in laminas 7 and 10, areas reported to receive afferents from viscera and muscles (Grant, 1995).

LSt neurons are ideally situated to relay ejaculation-related information to the mSPFp, and ejaculation results in neural activation of both LSt and mSPFp neurons. However, it is not clear whether activation of LSt neurons is causally related to activation of neurons in the mSPFp. In addition, it is unknown whether the neuropeptides localized in LSt neurons (galanin, CCK, and enkephalin) are involved in activation of mSPFp neurons. Finally, it is unknown whether other mSPFp-projecting cells (non-LSt) are activated with ejaculation. Other mSPFp-projecting neurons are primarily situated in L5-L6 (Greco et al., 1999) (Coolen, Veening, A. B. Wells, Shipley, unpublished observations). Indeed, male sexual behavior causes activation of neurons in L5 and L6, including neurons that project to the central tegmental field (Greco et al., 1998, 1999), which encompasses the mSPFp, as well as in mSPFp-projecting neurons (Coolen, Veening, Wells, Shipley, unpublished observations). However, Fos expression in L5 and L6 mSPFp-projecting cells may not be related to ejaculation, because we did not observe ejaculation-induced Fos expression in these spinal cord levels (Truitt and Coolen, unpublished observations). In addition, Fos expression in non-LSt cells in the L3-L4 is not specifically related to ejaculation. However, it should be noted that without identification of mSPFp-projecting neurons, ejaculation-specific activation is easily overlooked. In fact, Fos expression in LSt cells comprises only $2.8 \%$ of the total Fos population. Nonetheless, to date it is the only population of cells in L3 and L4 observed to be activated specifically with ejaculation.

We have argued that the activation of LSt neurons in males is a consequence of ejaculation. Subsequently, activation of these neurons could trigger ejaculation. Indeed, we demonstrated recently that selective lesions of LSt cells completely disrupted ejaculatory behavior while not effecting other components of male sexual behavior (Truitt and Coolen, 2002). The pathways via which LSt neurons may be crucially involved in ejaculation are speculative and may include local projections to preganglionic or motor neurons controlling muscles associated with emission or ejaculation, or supraspinal projections to brain regions that in turn can control ejaculation. In addition, descending inputs may activate LSt cells. Whether LSt neurons receive direct or indirect descending inputs from brain regions controlling ejaculation is currently unclear. Additional studies investigating activation of LSt neurons after ejaculation in spinalized animals will be needed to address this issue.

In contrast to the robust LSt cell activation with ejaculation in male rats, LSt cells were not activated with any component of female sexual behavior. It is curious that vaginocervical stimulation during sexual behavior in female rats induces Fos expression in the mSPFp (Coolen et al., 1996, 1997a) but not in LSt neurons. Thus, it is possible that information related to female sexual function is relayed to the mSPFp and other areas of the brain via pathways that do not include the LSt neurons. Obviously, based on a lack of Fos expression it cannot be concluded that these LSt neurons are not involved with sexual behavior in the female, and other markers of activation may indeed demonstrate that these neurons are activated after female sexual behavior. A potential alternate pathway may consist of mSPFp-projecting neurons in 
L5-L6, where Fos is indeed induced by vaginocervical stimulation (Lee and Erskine, 1996, 2000; Truitt and Coolen, unpublished observations). However, the location of mSPFp-projecting neurons other than LSt cells in females is currently unknown. Nonetheless, the current data illustrate a sexual dimorphic activation of LSt cells during male and female sexual behavior.

In conclusion, in the current study we identified a group of spinothalamic neurons that are selectively activated with ejaculation. LSt neurons are activated specifically when copulation includes ejaculation, but not with other aspects of male sexual behavior. Moreover, vaginocervical stimulation during mating was not sufficient to induce Fos expression in the LSt neurons of female rats. We hypothesize that LSt cells are in the position to process and relay information related to ejaculation to the thalamus (mSPFp), and may thus contribute to the sensory sensation and rewarding properties of ejaculation. Together, these data provide novel insights into the function of spinothalamic neurons and contribute to our understanding of neural pathways related to sexual function.

\section{References}

Ahlenius S, Larsson K, Svensson L, Hjorth S, Carlsson A, Lindberg P, Wikstrom H, Sanchez D, Arvidsson L, Hacksell U, Nilsson J (1981) Effects of a new type of 5-HT receptor agonist on male rat sexual behavior. Pharmacol Biochem Behav 15:785-792.

Coolen LM, Peters HJ, Veening JG (1996) Fos immunoreactivity in the rat brain following consummatory elements of sexual behavior: a sex comparison. Brain Res 738:67-82.

Coolen LM, Peters HJ, Veening JG (1997a) Distribution of Fos immunoreactivity following mating versus anogenital investigation in the male rat brain. Neuroscience 77:1151-1161.

Coolen LM, Olivier B, Peters HJ, Veening JG (1997b) Demonstration of ejaculation-induced neural activity in the male rat brain using 5-HT1A agonist 8-OH-DPAT. Physiol Behav 62:881-891.

Coolen LM, Peters HJ, Veening JG (1998) Anatomical interrelationships of the medial preoptic area and other brain regions activated following male sexual behavior: a combined fos and tract-tracing study. J Comp Neurol 397:421-435.

DeGroat W, Steers W (1990) Autonomic regulation of the urinary bladder and sexual organs. In: Central regulation of autonomic functions (Loewy A, Spyer K, eds), pp 310-330. New York: Oxford UP.

Erskine MS (1993) Mating-induced increases in FOS protein in preoptic area and medial amygdala of cycling female rats. Brain Res Bull 32:447-451.

Grant G (1995) Primary afferent projections to the spinal cord. In: The rat nervous system, Ed 2 (Paxinos G, ed), pp 61-66. San Diego: Academic.

Greco B, Edwards DA, Zumpe D, Michael RP, Clancy AN (1998) Fos induced by mating or noncontact sociosexual interaction is colocalized with androgen receptors in neurons within the forebrain, midbrain, and lumbosacral spinal cord of male rats. Horm Behav 33:125-138.

Greco B, Edwards DA, Michael RP, Zumpe D, Clancy AN (1999) Colocalization of androgen receptors and mating-induced FOS immunoreactivity in neurons that project to the central tegmental field in male rats. J Comp Neurol 408:220-236.

Heeb MM, Yahr P (1996) c-Fos immunoreactivity in the sexually dimorphic area of the hypothalamus and related brain regions of male gerbils after exposure to sex-related stimuli or performance of specific sexual behaviors. Neuroscience 72:1049-1071.

Ju G, Melander T, Ceccatelli S, Hokfelt T, Frey P (1987) Immunohistochemical evidence for a spinothalamic pathway co-containing cholecystokinin- and galanin-like immunoreactivities in the rat. Neuroscience 20:439-456.

Kollack-Walker S, Newman SW (1995) Mating and agonistic behavior produce different patterns of Fos immunolabeling in the male Syrian hamster brain. Neuroscience 66:721-736.

Kollack-Walker S, Newman SW (1997) Mating-induced expression of c-fos in the male Syrian hamster brain: role of experience, pheromones, and ejaculations. J Neurobiol 32:481-501.

Lee JW, Erskine MS (1996) Vaginocervical stimulation suppresses the expression of c-fos induced by mating in thoracic, lumbar, and sacral segments of the female rat. Neuroscience 74:237-249.

Lee JW, Erskine MS (2000) Changes in pain threshold and lumbar spinal cord immediate-early gene expression induced by paced and nonpaced mating in female rats. Brain Res 861:26-36.

Meisel R, Sachs B (1994) The physiology of male sexual behavior. In: The physiology of reproduction, Ed 2 (Knobil E, Neill J, eds), pp 3-105. New York: Raven.

Nadelhaft I, Booth AM (1984) The location and morphology of preganglionic neurons and the distribution of visceral afferents from the rat pelvic nerve: a horseradish peroxidase study. J Comp Neurol 226:238-245.

Newman HF, Reiss H, Northup JD (1982) Physical basis of emission, ejaculation, and orgasm in the male. Urology 19:341-350.

Newman S, Parfitt D, Kollack-Walker S (1997) Mating-induced $c$-fos expression patterns complement and supplement observations after lesions in the male Syrian hamster brain. Ann NY Acad Sci 807:239-259.

Nicholas AP, Zhang X, Hokfelt T (1999) An immunohistochemical investigation of the opioid cell column in lamina $\mathrm{X}$ of the male rat lumbosacral spinal cord. Neurosci Lett 270:9-12.

Paredes RG, Vazquez B (1999) What do female rats like about sex? Paced mating. Behav Brain Res 105:117-127.

Paxinos G, Watson C (1998) The rat brain in stereotaxic coordinates, Ed 4. San Diego: Academic.

Pfaus JG, Heeb MM (1997) Implications of immediate-early gene induction in the brain following sexual stimulation of female and male rodents. Brain Res Bull 44:397-407.

Pfaus JG, Kleopoulos SP, Mobbs CV, Gibbs RB, Pfaff DW (1993) Sexual stimulation activates $\mathrm{c}$-fos within estrogen-concentrating regions of the female rat forebrain. Brain Res 624:253-267.

Polston EK, Erskine MS (2001) Excitotoxic lesions of the medial amygdala differentially disrupt prolactin secretory responses in cycling and mated female rats. J Neuroendocrinol 13:13-21.

Rowe DW, Erskine MS (1993) c-Fos proto-oncogene activity induced by mating in the preoptic area, hypothalamus, and amygdala in the female rat: role of afferent input via the pelvic nerve. Brain Res 621:25-34.

Tetel MJ, Getzinger MJ, Blaustein JD (1993) Fos expression in the rat brain following vaginal-cervical stimulation by mating and manual probing. J Neuroendocrinol 5:397-404.

Tracey D (1995) Ascending and descending pathways in the spinal cord. In: The rat nervous system, Ed 2 (Paxinos G, ed), pp 67-80. San Diego: Academic.

Truitt WA, Coolen LM (2002) Identification of a potential ejaculation generator in the spinal cord. Science 297:1566-1569.

Veening JG, Coolen LM (1998) Neural activation following sexual behavior in the male and female rat brain. Behav Brain Res 92:181-193. 